\title{
GRAIN SPECTROSCOPY
}

\author{
L.J. ALLAMANDOLA \\ NASA-Ames Research Center \\ Mountain View, CA 94035 \\ USA
}

\section{Introduction}

Our fundamental knowledge of interstellar grain composition has grown substantially during the past two decades thanks to significant advances in two areas: astronomical infrared spectroscopy and laboratory astrophysics. The opening of the mid-infrared, the spectral range from $4000-400 \mathrm{~cm}^{-1}(2.5-25 \mu \mathrm{m})$, to spectroscopic study has been critical to this progress because spectroscopy in this region reveals more about a material's molecular composition and structure than any other physical property.

Infrared spectra which are diagnostic of interstellar grain composition fall into two categories: absorption spectra of the dense and diffuse interstellar media, and emission spectra from UV-Vis rich dusty regions. The former will be presented in some detail, with the later only very briefly mentioned. This paper summarizes what we have learned from these spectra and presents "doorway" references into the literature. Detailed reviews of many aspects of interstellar dust are given in [1]. Reviews of the IR spectra of dense clouds, emphasizing observations, can be found in [2,3] and those emphasizing laboratory and theoretical considerations in [4-9].

\section{Absorption Spectroscopy}

\subsection{DENSE INTERSTELLAR MEDIUM}

IR spectroscopy shows that dust in dense molecular clouds is comprised principally of mixed molecular ices and silicates. This conclusion is based on the direct comparison of astronomical spectra with the spectra of laboratory analogs as illustrated in Figure 1. The ice compositions and abundances derived from these spectra for several molecular clouds are summarized in Table 1. The major conclusions drawn from each spectral region comprising the mid-IR are discussed below.

2.1.1. The 4000-2500 $\mathrm{cm}^{-1}(2.5-4 \mu \mathrm{m})$ Region. The deep $3250 \mathrm{~cm}^{-1}(3.08 \mu \mathrm{m}) \mathrm{band}$, a common characteristic of molecular cloud spectra, is attributed to the $\mathrm{OH}$ stretch in frozen $\mathrm{H}_{2} \mathrm{O}$, the most abundant interstellar ice constituent known. Small contributions from O-H (alcoholic) and $\mathrm{N}-\mathrm{H}$ (amine) stretches are also likely. In dense clouds this band has a low frequency wing, [10-14] in contrast to the more symmetric profile associated with OH IR stars $[15,16]$. The wing, which extends from approximately 3000 to $2700 \mathrm{~cm}^{-1}(3.3$ to $3.7 \mu \mathrm{m})$, has been attributed to $\mathrm{H}_{2} \mathrm{O}$-base interactions, hydrocarbons, and scattering [4, $17,18]$. Spectral observations tend to support the first two $[13,10]$ while polarization studies seem marginally inconsistent with the latter [19]. Theoretical discussions of this band are in references $[17,4,20]$.

Spectral features associated with aliphatic hydrocarbon C-H stretches were long expected on the wing [4] and have recently been found. The importance of methanol [5] has been confirmed by detection of a band at $2825 \mathrm{~cm}^{-1}(3.5 \mu \mathrm{m})[21,14]$. When present, $\mathrm{CH}_{3} \mathrm{OH}$ is the second most abundant ice constituent $\left(\sim 10-50 \%\right.$ wrt $\left.\mathrm{H}_{2} \mathrm{O}\right)$. A broader feature peaking near $2880 \mathrm{~cm}^{-1}(3.4 \mu \mathrm{m})$ has also been found. This frequency is 


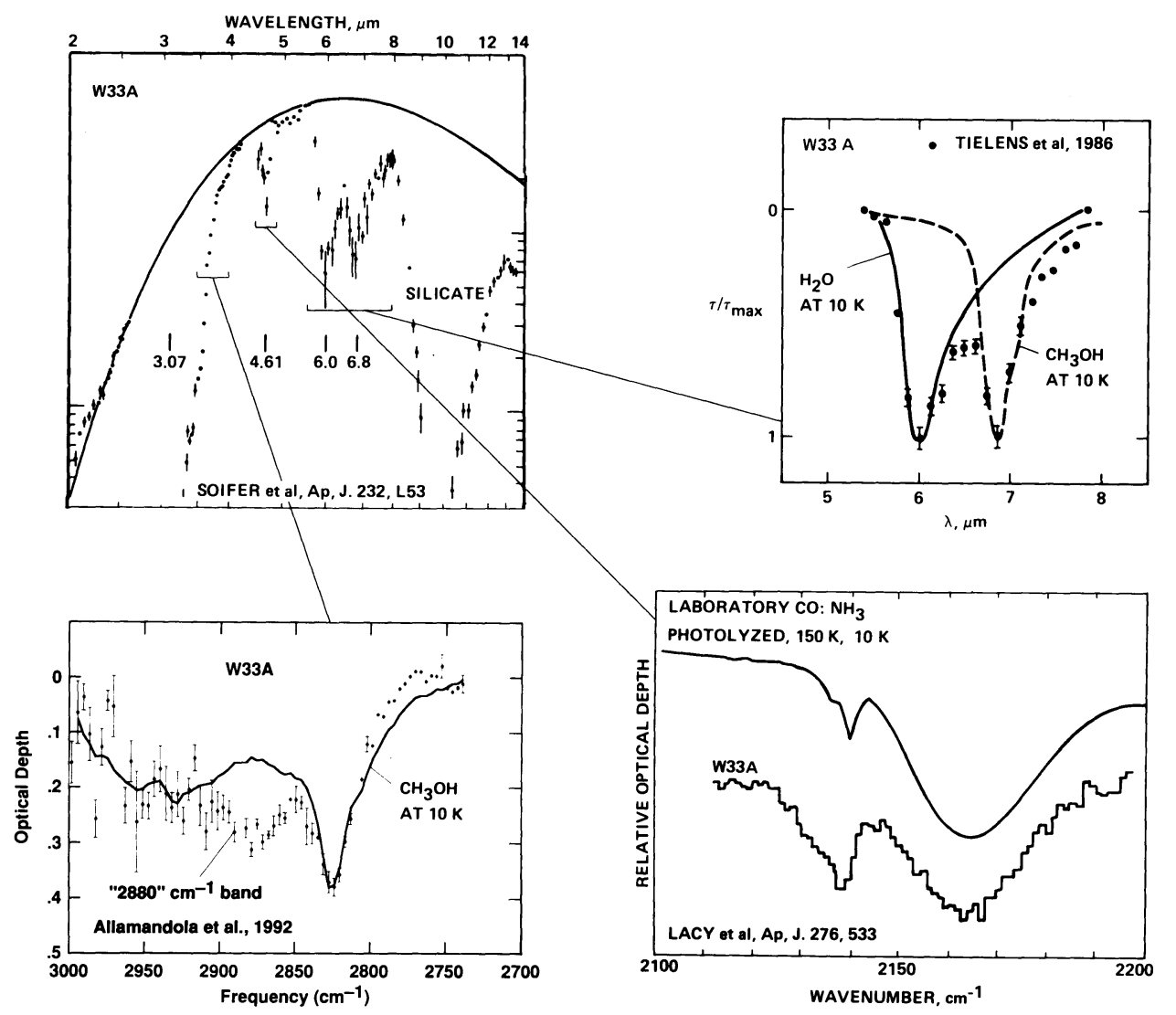

Figure 1. The mid-infrared spectrum of W33A compared with spectra of laboratory analogs.

characteristic of $\mathrm{C}-\mathrm{H}$ stretching vibrations for $\mathrm{H}$ on "tertiary carbon", i.e. aliphatic carbon atoms which are bound to three other carbon atoms and one hydrogen atom. The dominance of this feature over primary and secondary $\mathrm{CH}$ stretching features suggests that a material with a diamond-like carbon skeleton is ubiquitous in dense clouds [14].

2.1.2 The 2500-2000 $\mathrm{cm}^{-1}$ (4-5 $\left.\mu \mathrm{m}\right)$ Region. Frozen CO is responsible for the band near $2140 \mathrm{~cm}^{-1}$ found in many, but not all, clouds [22-24]. The peak position of this band shows that the solid $\mathrm{CO}$ is distributed between two forms of interstellar ice, one dominated by $\mathrm{H}_{2} \mathrm{O}$, the other by some non-polar material $[25,26]$. When $\mathrm{CO}$ is present, the $\mathrm{CO} / \mathrm{H}_{2} \mathrm{O}$ concentration of interstellar ices is typically about a few percent.

The broad feature near $2165 \mathrm{~cm}^{-1}(4.6 \mu \mathrm{m})$ in Figure 1 has also been detected in one or two other objects (Tielens, Teglar-private communications). Laboratory experiments show that the peak position and profile indicate a $\mathrm{CN}$ stretch [22]. The initial assignment to a nitrile $(-C \equiv N)$ or iso-nitrile $(C=N-)$ has recently been questioned. The $C N$ stretch in the $\mathrm{OCN}^{-}$ion [27], and the Si-H stretch $[28,29]$ have been suggested in its place. In the author's opinion, the initial assignment remains the most attractive. Further experiments are needed to settle this. 
A weak feature at $2040 \mathrm{~cm}^{-1}(4.9 \mu \mathrm{m})$ in the spectrum of W33A suggests that OCS is also.an interstellar ice constituent [30].

2.1.3 The 2000-1000 $\mathrm{cm}^{-1}(5-10 \mu \mathrm{m})$ Region. The main ice bands here peak near 1670 and $1470 \mathrm{~cm}^{-1}(6.0$ and $6.8 \mu \mathrm{m})$. These are evident in several clouds and are fit quite well by $\mathrm{H}_{2} \mathrm{O}\left(1670 \mathrm{~cm}^{-1}\right)$ and $\mathrm{CH}_{3} \mathrm{OH}\left(1670 \mathrm{~cm}^{-1}\right)$ [5].

The column densities of both $\mathrm{H}_{2} \mathrm{O}$ and $\mathrm{CH}_{3} \mathrm{OH}$ derived from these bands exceeds those derived from their corresponding $\mathrm{OH}$ and $\mathrm{CH}$ stretches near $3000 \mathrm{~cm}^{-1}$ (Table 1). This inconsistency can be rationalized in the following ways: 1 - Other species may contribute significantly to the $1470 \mathrm{~cm}^{-1}(6.8 \mu \mathrm{m})$ band [21]. $\mathrm{NH}_{4}^{+}$has been suggested [31]. However, if this were the case, clearly resolved features which correspond to the $\mathrm{NH}$ stretching vibrations should be evident in the $3500-3000 \mathrm{~cm}^{-1}(2.8-3.3 \mu \mathrm{m})$ region ; 2The $2825 \mathrm{~cm}^{-1} \mathrm{CH}$ stretch blends with other features and its optical depth is a lower limit [14]; and 3- Higher frequency $\left(3000 \mathrm{~cm}^{-1}, 3 \mu \mathrm{m}\right)$ radiation reaching the telescope includes scattered light which fills in the absorptions in this region whereas lower frequency radiation $\left(<2000 \mathrm{~cm}^{-1} ;>5 \mu \mathrm{m}\right)$ does not $[18,32]$.

Recently, weak features in the $1400-1250 \mathrm{~cm}^{-1}(7-8 \mu \mathrm{m})$ region have been detected [33]. That at $1301 \mathrm{~cm}^{-1}(7.685 \mu \mathrm{m})$ is attributed to methane, implying rather low solid state abundances for this molecule. Frozen $\mathrm{CH}_{4}$ appears to be approximately 100 times less abundant than solid $\mathrm{CO}$ [33] which in turn is typically a few percent the abundance of $\mathrm{H}_{2} \mathrm{O}$ ice.

2.1.4 The 1000-500 $\mathrm{cm}^{-1}(10-20 \mu \mathrm{m})$ Region. This region is dominated by the broad "SiO" stretch near $1000 \mathrm{~cm}^{-1}(10 \mu \mathrm{m})[2,3,34]$. This interstellar material had been thought to be amorphous due to the lack of spectral structure on the band. However, recent higher resolution spectroscopic and polarization measurements imply that crystalline material is also present $[35,36]$.

As with the ices, laboratory studies give important insight into the molecular nature of this interstellar refractory material [37-43]. Laboratory experiments show that the band profile reveals much about the material's formation conditions and subsequent processing $[40,42,43]$. Unfortunately, although this feature was extensively studied in the 70's , high resolution spectroscopic studies utilyzing modern astronomical instruments have not been carried out. In view of the wealth of information one can extract from such studies, it is hoped that a significant amount of observing time with the new generation of astronomical, high resolution, IR spectrometers is devoted to measuring this feature, looking specifically for substructure. In particular, crystalline silicates can produce resolvable structure near $890 \mathrm{~cm}^{-1}(11.2 \mu \mathrm{m})$. Furthermore, space-borne spectrometers will provide the frequency, strength, and profile of the bending modes near $400 \mathrm{~cm}^{-1}$ (20 $\mu \mathrm{m})$. Such complete coverage can provide important insight into the interstellar material's history [36,43] and degree of crystallinity.

In addition to these features which have long been known to be associated with refractories, absorptions due to ice constituents have recently been reported in this region. There is good evidence for the librational band of $\mathrm{H}_{2} \mathrm{O}$ (and probably some alcohols) at 830 $\mathrm{cm}^{-1}(12 \mu \mathrm{m})$ [44] and the $667 \mathrm{~cm}^{-1}(15 \mu \mathrm{m})$ band of $\mathrm{CO}_{2}$ [45] in IRAS-LRS spectra of a few clouds. For the objects studied, the solid $\mathrm{CO}_{2}$ abundance seems comparable to that of solid CO. 
Table 1. Column densities of some molecular cloud dust components.

\begin{tabular}{|c|c|c|c|c|}
\hline \multirow[t]{2}{*}{ MATERIAL } & \multicolumn{4}{|c|}{ OBJECT } \\
\hline & NGC 7538 IRS 9 & W33A & W3 IRS 5 & $\underline{\text { S140 IRS } 1}$ \\
\hline Hydrogen_a & $1.6 \times 10^{23}$ & $2.8 \times 10^{23}$ & $2.7 \times 10^{23}$ & $1.4 \times 10^{23}$ \\
\hline \multicolumn{5}{|l|}{$\mathrm{H}_{2} \mathrm{O}$} \\
\hline $3250 \mathrm{~cm}^{-1} \mathrm{~d}$ & $>5.3 \times 10^{18}$ & $>8.6 \times 10^{18}$ & $3.7 \times 10^{18}$ & $2.1 \times 10^{18}$ \\
\hline $1650 \mathrm{~cm}^{-1 ~ d}$ & $1.1 \times 10^{19}$ & $4.2 \times 10^{19}$ & $4.3 \times 10^{18}$ & $8.8 \times 10^{18}$ \\
\hline \multicolumn{5}{|l|}{$\mathrm{CH}_{3} \mathrm{OH}$} \\
\hline $2825 \mathrm{~cm}^{-1 ~ b}$ & $9.1 \times 10^{17}$ & $3.9 \times 10^{18}$ & $<5.3 \times 10^{17}$ & $<3.8 \times 10^{17}$ \\
\hline $2600+2540 \mathrm{~cm}^{-1} \mathrm{c}$ & $\cdots$ & $2.5 \times 10^{18}$ & $\cdots$ & $\cdots$ \\
\hline $1470 \mathrm{~cm}^{-1 ~ d}$ & $7.1 \times 10^{18}$ & $2.3 \times 10^{19}$ & $3.5 \times 10^{18}$ & $\cdots$ \\
\hline $\mathrm{CO}$ (non-polar ice) $\mathrm{e}^{\mathrm{a}}$ & $6.4 \times 10^{17}$ & $1.1 \times 10^{17}$ & $1.1 \times 10^{17}$ & $\cdots$ \\
\hline $\mathrm{CO}(\text { polar ice })^{e}$ & $3.2 \times 10^{17}$ & $2.8 \times 10^{17}$ & $5.4 \times 10^{16}$ & $\cdots$ \\
\hline $\mathrm{CO}$ (gas) $\mathrm{e}$ & $1.4 \times 10^{19}$ & $2.0 \times 10^{19}$ & $2.2 \times 10^{19}$ & $\cdots$ \\
\hline "Diamonds"b & $3.8 \times 10^{18}$ & $1.5 \times 10^{18}$ & $2.8 \times 10^{18}$ & $2.8 \times 10^{18}$ \\
\hline
\end{tabular}

a)From $\mathrm{N}_{H}=1.9 \times 10^{21} \mathrm{~A}_{\mathrm{v}}$; b), c) [14]; d) [5], except for S140 IRS 1 from [2]; e) [26].

\subsection{DIFFUSE INTERSTELLAR MEDIUM}

Mid-IR spectra of the diffuse ISM are dominated by the SiO stretch. As mentioned above, high resolution spectroscopic studies of this feature, searching for substructure, are very important to carry out.

Most conclusions regarding other diffuse medium dust components derived from IR spectroscopy are based on studies in the OH/NH/CH stretch region $\left(3500-2700 \mathrm{~cm}^{-1} ; 2.9\right.$ $3.7 \mu \mathrm{m}$ ). These studies have focused on Galactic Center sources, particularly infrared source number 7 (GC IRS7) [46-49]. This work has recently been expanded to include VI Cyg 12 and several other objects $[50,51,52]$.

Figure 2 shows that there is a reasonable match between the spectrum of GC IRS7 and a laboratory residue produced by the vacuum-UV irradiation of an interstellar ice analog [51]. The match between the interstellar feature and the C-H stretch band in the spectrum of hydrocarbons sublimed from the Murchison meteorite is remarkable [52]. Based on these observations it appears that the aliphatic component of diffuse medium dust has a $-\mathrm{CH}_{2}-/-\mathrm{CH}_{3}$ ratio of about 2, with chains containing 3-4 carbon atoms that have one end attached to an electronegative group such as $-\mathrm{OH},-\mathrm{C} \equiv \mathrm{N}$, or perhaps an aromatic network. About $8-20 \%$ of cosmic carbon is tied up in this component. 


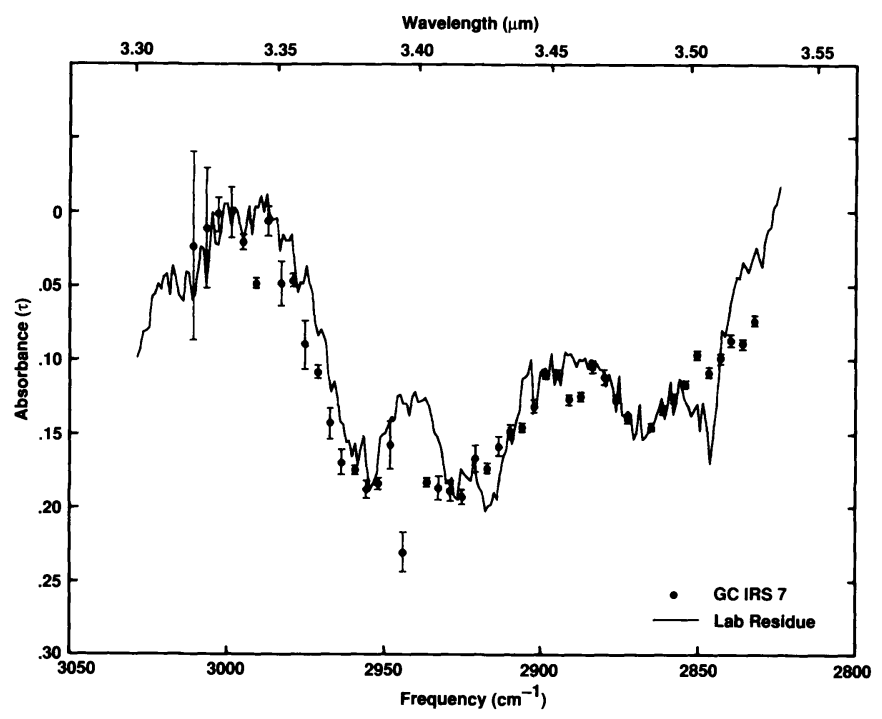

Figure 2. The spectrum, in the $\mathrm{CH}$ stretch region, of Galactic Center source IRS 7 compared to that of a residue produced by the irradiation of an interstellar ice analog.

\section{Emission Spectroscopy}

A family of infrared emission bands from $3050-870 \mathrm{~cm}^{-1}(3.28-11.5 \mu \mathrm{m})$ carry a significant fraction of the energy radiated by many different objects in which an intense UV-Vis radiation field is attenuated by dust. An example of a typical spectrum is given in Figure 3. The "resemblance" of this spectrum to spectra of polycyclic aromatic hydrocarbons (PAHs) and related materials such as amorphous carbon (HAC) has been taken as compelling evidence that these materials are responsible. Analysis of these midinfrared emission spectra indicates that a few percent of the cosmic carbon is tied up in the small (20-40 carbon atom) PAHs which are responsible for the sharp IR features, and a similar amount is tied up in the larger (200-500 carbon atom) PAHs, PAH clusters, and amorphous carbon particles responsible for the underlying broad spectral components.

The aromatic nature of the carrier seems secure. However, there is still some debate as to whether or not the emitters of the discrete features are free molecular PAHs, or PAH units which make up HAC or other carbonaceous material. All this and more can be found in the following extensive reviews and papers: Molecular PAHs: [53,54] HAC: [55,56,57]; QCC: [58,59]. 

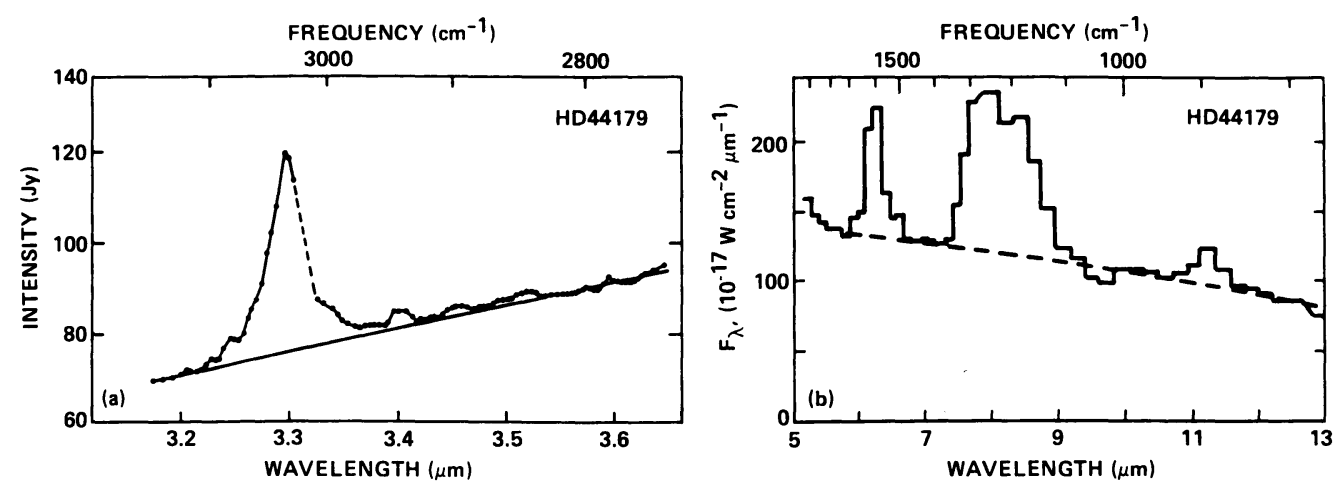

Figure 3. The spectrum of HD 44179 showing the family of emission bands associated with PAHs. These features are emitted from many different astronomical objects.

\section{References}

1. Allamandola, L.J. and Tielens, A.G.G.M.(1989) eds.Interstellar Dust: IAU Symposium 135, (Kluwer, Dordrecht).

2. Willner, S.P., Gillett, F.C., Herter, T.L., Jones, B., Krassner, J., Merrill, K.M., Pipher, J.L., Puetter, R.C., Rudy, R.J., Russell, R.W., and Soifer, B.T.(1982) Ap.J., 253, 174.

3. Whittet, D.C.B.(1988) in Bailey, M.E. and Williams, D.A. (eds.), Dust in the Universe, Cambridge Univ. Press, Cambridge, 25-54.

4. Allamandola, L.J.(1984) in Kessler, M.F. and Phillips, J.P. (eds.), Galactic and Extragalactic Infrared Spectroscopy; Reidel, Dordrecht, 5-35.

5. Tielens, A.G.G.M., and Allamandola, L.J. (1987) in Morfill, G.E. and Scholer, M., (eds.), Physical Processes in Interstellar Clouds, D. Reidel, Dordrecht, 333-376.

6. Tielens, A.G.G.M., and Allamandola, L.J. (1987) in Hollenbach, D.J. and Thronson, H. A. (eds.) Interstellar Processes, D. Reidel, Dordrecht, 397-469.

7. Allamandola, L.J. and Sandford, S.A. (1988) in Bailey, M.E. and Williams, D.A. (eds.), Dust in the Universe, Cambridge Univ. Press, Cambridge, 229-264.

8. Tielens, A.G.G.M. (1989) in Allamandola, L.J., and Tielens, A.G.G.M. (eds.) Interstellar Dust:, Kluwer, Dordrecht, 239-262.

9. Bussoletti, E. and Colangeli, L. (1990) Rivista del Nuovo Cimento 13, 1-70

10. Smith, R.G., Sellgren, K., and Tokunaga, A.T., (1989) Ap.J. 344, 413.

11. Nagata T., Tokunaga, A. T., Sellgren, K., Smith, R.G., Onaka, T., Nakada, Y., and Sakata, A. (1988) Ap. J. (Letters) 287, L51.

12. Whittet, D.C.B., Bode, M.F., Longmore, A.J., Baines, D.W.T., and Evans, A. (1983) Nature 303, 218-221. 
13. Eiroa, C. and Hodapp, K.W. (1989) A\&A, 210, 345-350

14. Allamandola, L.J., Sandford, S.A., Tielens, A.G.G.M., and Herbst, T. (1992) Ap.J. in press

15. Soifer, B.T., Willner, S.P., Capps, R.W., and Rudy, R.J. (1981) Ap. J. 250,631.

16. Smith, R.G., Sellgren, K., and Tokunaga, A.T.(1987) Ap.J. 334, 209-219.

17. Tielens, A.G.G.M., and Hagen, W.(1982) A\&A 114, 245.

18. Leger, A., Gauthier, S., Defourneau, D., and Rouan, D. (1983) A\&A 117, 164-169.

19. Hough, J.H. et al., (1988) MNRAS 230, 107.

20. Greenberg, J.M., van de Bult, C.E.P.M., and Allamandola, L.J. (1983) J. Phys. Chem. 87, 4243.

21. Grim, R.J.A., Baas, F., Geballe, T.R., Greenberg, J.M., and Schutte, W. (1991) A\&A, 243, 473-477

22. Lacy, J.H., Baas, F., Allamandola, L.J., Persson, S.E., McGregor, P.J., Lonsdale, C.J., Geballe, T.R., and van de Bult, C.E.P.M. (1984) Ap. J. 276, 533-543.

23. Geballe, T.R. (1986) A\&A 162, 248-252.

24. Whittet, D.C.B., Adamson, A.J., Duley, W.W., Geballe, T.R., and McFadzean, A.D. (1989) MNRAS 241, 707-720.

25. Sandford, S.A., Allamandola, L.J., Tielens, A.G.G.M., and Valero, G.J. (1988) Ap.J. 329, 498-510.

26. Tielens, A.G.G.M., Tokunaga, A.T., Geballe, T.R., and Baas, F. (1991) Ap. J. 381, 181-199.

27. Grim, R., and Greenberg, J.M. (1987) Ap. J. (Letters) 321, L91.

28. Nuth, J.A., and Moore, M.H. (1988)Ap. J. (Letters) 329, L113.

29. Moore, M.H., Tanabe, T., and Nuth, J.A. (1991) Ap. J. (Letters) 373, L31-L34

30. Geballe, T.R., Baas, F., Greenberg, J.M., and Schutte, W. (1985) A\&A 146 L6-L8.

31. Grim, R.J.A., Greenberg, J.M., Schutte, W.A., and Schmitt, B.(1989) Ap. J. (Letters) 241, L87.

32 Pendleton, Y., Tielens, A.G.G.M., and Werner, M.W. (1990) Ap.J. 349, 107.

33. Lacy, J.H., Carr, J.S., Evans II, N.J., Baas, F., Achtermann, J.M., and Arens, J.F.(1991) Ap.J. 376, 556-560.

34. Merrill, K.M. and Stein, W.A. (1976) PASP 88, 874-887.

35. Aitken, D.K., Roche, P., Smith, C.H., James, S.D., and Hough, J.H. (1988) MNRAS 230, 629-638.

36 Aitken, D.K., Smith, C.H., and Roche, P. (1989) MNRAS 236, 919-927.

37. Day, K.L.(1979) Ap.J. 234, 158-161.

38. Knacke, R.F. and Kratschmer, W.(1980) A\&A 92, 281-288.

39. Koike, C., Hasegawa, H., Asada, N., and Hattori, T. (1981) Ast.\&Sp. Sci. Rev 79, $77-$ 85

40. Nuth, J.A. and Donn, B. (1983) J. Geophys. Res. 88, Supplement, A847-A852.

41. Nuth, J.A., and Hecht, J.H. (1990) Ast.\&Sp. Sci. 163, 79-94.

42. Mukai, T. and Koike, C. (1990) Icarus 87, 180-187.

43. Nuth, J.A. (1992) in Singh , P.D. and Almeida, (eds.) Astrochemistry of Cosmic Phenomena: IAU Symposium 150, Kluwer, Dordrecht, in press

44. Cox, P. (1990) A\&A 225, L1.

45. d'Hendecourt, L.B., and Jourdain de Muizon, M. (1990) A\&A 223, L5.

46. Wickramasinghe, D.T., and Allen, D.A. (1980) Nature 287, 518.

47. Jones, T.J., Hyland, A.R., and Allen, D.A. (1983) Mon. Not. R. Astr. Soc. 205. 187.

48. Butchart, I., McFadzean, A.D., Whittet, D.C.B., Geballe, T.R., and Greenberg, J.M. (1986) Astron. Astrophys. 154, L5.

49. McFadzean, A.D., Whittet, D.C.B., Longmore, A.J., Bode, M.F., and Adamson,

A.J. (1989) Mon. Not. R. Astr. Soc. 241, 873.

50. Adamson, A.J., Whittet, D.C.B., and Duley, W.W. (1990) Mon. Not. R. Astr. Soc. 243, 400. 
51. Sandford, S.A., Allamandola, L.J., Tielens, A.G.G.M., Sellgren, K., Tapia, M., and Pendleton, Y.( 1991) Ap.J. 371, 607-620.

52. Pendleton et al (1992) in preparation

53. Allamandola, L.J., Tielens, A.G.G.M., and Barker, J.R.(1989) Ap.J. Suppl., 71, 733-775.

54. Puget, J.L. and Leger, A. (1989) ARA\&A, 27, 161.

55. Duley, W.W.and Williams, D.A. (1988) MNRAS 231, 969-975.

56. Duley, W.W.and Williams, D.A. (1990) MNRAS 247, 647-650.

57. Blanco, A., Bussoletti, E., and Colangeli, L. (1988) Ap.J. 334, 875-882.

58. Sakata, A., Wada, S., Onaka, T., and Tokunaga, A.T. (1987) Ap. J. (Letters) 320, L63-L67.

59. Sakata, A., Wada, S., Onaka, T., and Tokunaga, A.T. (1990) Ap. J.353, 543.

\section{QUESTIONS AND ANSWERS}

B. Khare: Could you comment on the particle size distribution from the laboratory spectra?

L. Allamandola: Apart from the interstellar $3.09 \mu \mathrm{m} \mathrm{H}_{2} \mathrm{O}$ band, these bands don't have wings which would arise from scattering. Thus, these spectra don't give size information.

A. Leger: Comment- It is not correct to say that there is no scattering in the IR. Very nice polarization measurements by Capps et al. in Orion have shown unambiguously scattering at two different wavelengths and allow a size determination. Actually the size found is about $0.5 \mu \mathrm{m}$, definitely larger than in the diffuse medium (Rouan et al. 1987)

L. d'Hendecourt: Comment- The identification of the $3.4 \mu \mathrm{m}$ band towards IRS 7 is far from unique. Carbon extract from the Orguel meteorite shows, in this region, exactly the same spectral fit to IRS 7 as was shown during this talk to a hydrocarbon produced by ice irradiation (Ehrenfreund Contributed paper, this meeting). Yet, this Orguel material is mostly made up of aromatic material while your material is aliphatic. This may shed some light on the origin of this material which might originate directly from carbon stars (eg CRL 618), and not from the UV photolysis of dirty ices which may not produce aromatic structure.

L. Allamandola: I agree. It is important to stress that this absorption only probes the aliphatic component and gives no insight into what other types of hydrocarbons may be present. In this regard it is also important to remember that olefinic hydrocarbons absorb in a similar region to aromatics, and olefins are produced in irradiation experiments. Thus even if there were a match of the $3.3 \mu \mathrm{m}$ band, aromatics are not necessarily implied.

D. Williams: The new feature at $2880 \mathrm{~cm}^{-1}$ is attributed by you to "diamond'-like carbon. Please describe this structure.

L. Allamandola: The smallest structure possible that meets the spectral requirements must contain more than 30 , mainly $\mathrm{sp}^{3}$ hybridized, carbon atoms which are bound to each other and hydrogenated on the surface. The spectra allow for a few $-\mathrm{CH}_{3}$ and- $\mathrm{CH}_{2}$ edge groups. 